\title{
Action militante en évolution : comprendre l'évolution des controverses causées par la mise en place d'une infrastructure
}

\author{
Étienne Lyrette et Michel Trépanier \\ INRS-UCS
}

\section{Introduction}

$\Gamma^{9}$ implantation d'une infrastructure provoque bien souvent une levée de boucliers de la part de la population ou de différents groupes. Divers intervenants s'affrontent, discutent, négocient dans le but de protéger leurs intérêts (économiques, qualité de vie, etc.). Connu sous le terme générique de syndrome NIMBY (Not In My Back Yard - Pas dans ma cour), ce phénomène de résistance et d'opposition a fait l'objet de beaucoup d'attention aux niveaux médiatique, professionnel et de la recherche dans différents domaines s'intéressant à l'aménagement du territoire. Toutefois, comme le laisse entendre le présent article, les controverses sociales entourant la mise en place d'une infrastructure ont passablement évolué au cours des dernières décennies. Cette évolution vient remettre en question l'utilisation quasi systématique de «l'étiquette » NIMBY pour décrire tout mouvement de riverains s'impliquant dans un débat touchant à l'implantation d'un équipement collectif. Afin de mener à bien notre réflexion, nous proposons de faire un survol du NIMBY, de remettre en question la vision "classique " associée à ce syndrome et de terminer avec la présentation de mouvements d'opposition qui illustrent l'évolution des controverses. Nous voulons ainsi participer à la réflexion entourant les défis posés par l'implantation d'infrastructures dans une société où les groupes sociaux et d'intérêt se multiplient et évoluent très rapidement.

\section{Le syndrome NIMBY}

Une définition «classique» du syndrome NIMBY pourrait se lire comme suit : «[...] l'implantation d'é- quipements collectifs qui se heurtent à l'opposition des populations locales concernées pour cause de nuisances diverses $[\ldots]$ inacceptables pour elles, mais parfaitement acceptables partout ailleurs où ces mêmes nuisances ne pourraient les toucher directement $»^{1}$.

Le syndrome NIMBY peut donc, à première vue, être compris comme une crise entre l'intérêt collectif et l'intérêt individuel. Selon plusieurs, ce type de situation peut nuire à la protection de l'environnement ainsi qu'au développement économique d'un secteur. En effet, plusieurs équipements (site d'enfouissement sanitaire, ligne de transport d'électricité, usine de recyclage, parc éolien, etc.) sont souvent requis pour régler un problème environnemental ou combler un besoin de la population en général, peu ou pas relié aux préoccupations des riverains directement touchés par l'infrastructure. De plus, dans certains cas, l'implantation d'une infrastructure majeure peut entrainer la création d'emplois en plus de participer au développement économique de la région touchée. Le rejet d'un de ces projets pour des motifs à caractères individuels peut donc avoir des retombées négatives sur l'environnement et sur l'économie d'une région.

Dans le cas d'infrastructures potentiellement à haut risque (pour la santé, la biosphère, etc.), telle une centrale au gaz ou nucléaire, plusieurs analyses lient ce problème au nécessaire arbitrage entre des effets négatifs concentrés en un lieu spécifique et des bienfaits dispersés sur le territoire ${ }^{2}$. Guay mentionne d'ailleurs que « [...] la concentration locale des risques et la diffusion nationale des bénéfices est la cause essentielle du syndrome Pas dans ma cour $»^{3}$. Toutefois, d'un point de vue pratique, la notion d'intérêt collectif 
n'est pas quelque chose de fixe et d'immuable. Tout dépend du planificateur, des acteurs qui prennent la parole, de ceux qui décident de ne pas la prendre, des groupes qui participent aux réunions, etc. Ceci ouvre la porte à une remise en question des fondements de la vision « classique » du NIMBY.

\section{Une remise en question de la vision « classique » du NIMBY}

$\mathrm{Au}$ cours des dernières décennies, le syndrome NIMBY a été énormément utilisé à bon et à mauvais escient. La surutilisation du syndrome a mené à une banalisation du terme. Au lieu d'être une notion clairement définie utilisée à des fins d'analyse, le syndrome NIMBY est souvent employé pour discréditer les opposants à un projet. Ces dernières années, le syndrome a été utilisé pour expliquer la quasi-totalité des projets de nature collective qui se sont soldés par un échec ${ }^{4}$. Pourtant, il est assez peu constructif de considérer systématiquement les opposants comme des individualistes sans vision collective et des freins au développement collectif ${ }^{5}$. Certains, comme Lascoumes $^{6}$, ont clairement démontré que les regroupements d'opposition à un projet peuvent prendre plusieurs formes et « [...] qu'il semble abusif de réduire toutes ces associations à des organisations qui visent exclusivement la satisfaction d'intérêts privés ». En effet, au-delà des préoccupations individuelles apparentes, les groupes défavorables tendent de plus en plus vers une logique de «l'intérêt général local ». Cette opposition peut être considérée comme une opportunité d'élargir le débat à une plus grande partie de la communauté.

D'autres, comme Lake (1993), proposent que le protectionnisme local, incarné par le syndrome NIMBY, représente une menace non pas aux objectifs de la société, mais plutôt aux intérêts financiers des différents groupes de promoteurs. Autrement dit, les acteurs qui font la promotion d'un projet réussissent souvent à faire passer leur intérêt particulier pour un intérêt général; ce qui leur permet de stigmatiser les groupes d'opposants comme des individualistes ou encore des « obstacles » au bien-être collectif. Les promoteurs et les partisans utilisent bien souvent ce que Callon et Law $^{7}$ qualifient de «traduction argumentaire» pour faciliter l'acceptabilité du projet aux yeux de la société en général et rallier le plus grand nombre d'acteurs possibles à leur cause. Les opposants peuvent égale- ment utiliser la même tactique pour discréditer le projet et amasser des appuis. Cette pratique de discréditation est, selon Lake, particulièrement présente dans les communautés occidentales, plus particulièrement celles d'Amérique du Nord qui favorisent fortement l'accumulation de capital.

\section{Au-delà des préoccupations individuelles apparentes, les groupes défavorables tendent de plus en plus vers une logique de «l'intérêt général local ».}

Nous pouvons donc constater que les définitions «classiques » du syndrome NIMBY sont remises en question. Il est également possible de faire état de changements dans la nature même des contestations à travers différents exemples. À ce chapitre, le cas du village de Vinon-sur-Verdon, situé dans la vallée du Rhône en France, est assez évocateur. Ce village est situé à 7 kilomètres du Centre d'énergie atomique (CEA) de Cadarache, «[...] où s'expérimentent les technologies de pointe du nucléaire et où le réacteur mondial de fusion thermonucléaire ITER pourrait $s^{\prime}$ installer $»^{8}$. Contrairement à ce que pourrait laisser entendre la littérature «classique» du syndrome NIMBY, les résidents riverains vivent très bien avec l'infrastructure et y voient même plusieurs avantages pour leur communauté :

[...] la courbe démographique est passée de 810 habitants en 1954 à 3700 aujourd'hui, et l'inversion s'est faite à partir de 1962, date de création du CEA.

[...] les maisons du vieux village sont retapées, le collège est flambant neuf, les cafés restent animés et les petites entreprises de bâtiment prospèrent, comme les deux agences immobilières. D'ailleurs, un quart des foyers du village vivent directement du CEA, parmi lesquels le premier adjoint au maire. Quant à l'inquiétude devant les technologies expérimentées dans ce qui reste, selon l'expression du maire, " un monde à part », elle est à peu près absente.

Lors du débat public d'octobre 2001 à propos du projet de nouveau stockage des déchets, ce sont des militants des réseaux antinucléaires venus des alentours qui s'étaient exprimés. Des Vinonnais pré- 
sents avaient posé quelques questions sur la pollution de l'eau, mais ce n'était pas allé beaucoup plus loin. $^{9}$

Comme le laisse voir la dernière citation, l'opposition au développement des installations de stockage de déchets nucléaires de la CEA n'est pas venue des riverains, mais bien de groupes environnementaux régionaux et nationaux :

Le réseau Sortir du nucléaire, revendiquant 650 associations membres, demande «l'annulation du projet de construction » à Cadarache. [...] Motif : la sismicité du sous-sol provençal.

Avec la sismicité, les associations antinucléaires espèrent trouver un défaut à la cuirasse d'un réacteur dont ils rejettent le principe, qu'il soit construit à Cadarache ou « à tout autre endroit. ${ }^{10}$

L'opposition n'est donc pas le lot des riverains mais bien de groupes externes. Ceci vient contredire la vision généralement acceptée du syndrome NIMBY. Cette tendance est également repérable au Québec. À cet effet, le cas le cas de la route 175 reliant la région du Saguenay-Lac-Saint-Jean à la ville de Québec est assez évocateur, alors que les principaux lobbies favorables à la mise en place de l'infrastructure étaient issus du milieu principalement touché ${ }^{11}$.

En résumé, il est clair que les définitions «classiques » du syndrome NIMBY et la vision des dynamiques d'implantation qui découlent de cette dernière sont contestées et semblent de moins en moins tenir la route. Nous ne remettons pas en question la définition même du NIMBY, mais bien la surutilisation du terme. Cette surutilisation en fait un « outil» conceptuel mal défini et mal adapté aux situations qu'il devrait aider à saisir. D'autres avenues doivent être envisagées afin de comprendre plusieurs controverses qui entourent la mise en place d'une infrastructure.

\section{Pas dans ma cour et nulle part ailleurs... une évolution du NIMBY?}

Est-ce que l'opposition face à l'implantation d'équipement a évolué au cours des dernières décennies? Est-ce que cette évolution expliquerait, en partie, le fait que le syndrome NIMBY semble de moins en moins « applicable » ? La montée de groupes d'oppo-

sition un peu plus « radicaux » qui s'entremêlent aux groupes d'opposition plus « traditionnels » peut venir, du moins en partie, répondre à ces interrogations et expliquer certains des changements apparents dans les dynamiques sociales qui entourent les controverses d'implantation. "Parfois, l'analyse de ces mouvements d'opposition est ambivalente, car s'y entremêlent en proportion variable des opposants NIMBY égoïstement centrés sur leur propres intérêts et des radicaux qui prônent un inconditionnel "Ni ici, ni ailleurs" plus critique et altruiste $\gg{ }^{12}$.

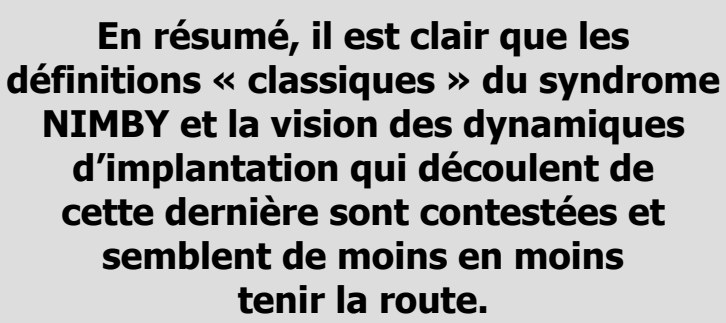

Ces changements dans les caractéristiques et les pratiques des groupes d'opposition sont à l'origine du développement d'une nouvelle terminologie. Pour Retzinger, les nouveaux termes comme NOPE (Not on Planet Earth - Pas sur la planète Terre), BANANA (Build Absolutely Nothing Anywhere Near Anybody - Ne rien construire nulle part ni près de qui que ce soit) ou encore NIABY (Not In Anybody's Backyard - Dans la cour de personne) sont un « raffinement » du NIMBY qui ont pour objectif de décrire de nouvelles réalités ${ }^{13}$. Pour d'autres, comme Greenburg et Osafo ${ }^{14}$ ces changements terminologiques sont carrément le reflet d'une évolution du syndrome NIMBY qui se serait amorcée dès les années 1980.

Dans un premier temps, nous devons comprendre que ces nouveaux termes expriment une radicalisation des débats et de l'opposition entourant la mise en place d'une infrastructure. De plus, il nous est possible de constater que cette radicalisation est liée à une perte d'importance de la localisation de l'équipement. En effet, le lieu n'a plus d'importance alors que les opposants sont contre l'implantation d'un type d'infrastructure sur l'ensemble du territoire. Cette évolution du syndrome NIMBY pourrait être qualifiée de radicalisation sans assise spatiale.

Afin de mieux cerner ce phénomène, nous allons examiner brièvement le projet de site d'entreposage de 
déchets nucléaires permanent à Yucca Mountain aux États-Unis. Le site envisagé pour accueillir le projet (entrée en opération prévue pour 2020) se trouve au Nevada, sur des terres fédérales, dans une région très isolée. Même si, physiquement, ce site est dans «la cour de personne », son choix soulève pourtant une vive opposition que Lobdell ${ }^{15}$ qualifie de typiquement NIMBY. L'opposition au choix du site de Yucca Mountain a su se rallier une foule d'acteurs très influents comme des journalistes et plusieurs politiciens de l'État et des États voisins. Avec l'aide des médias et de pressions politiques, ces acteurs sont parvenus à faire de Yucca Mountain un site à première vue reculé et ignoré de la majorité, la cour de l'ensemble des habitants de l'État. Toutefois, ces acteurs ne s'opposent pas à la mise en place d'un site permanent de stockage de déchets irradiés, ils remettent surtout en question la validité du site, ce qui est typique au NIMBY.

\section{Cette évolution du syndrome NIMBY pourrait être qualifiée de radicalisation sans assise spatiale.}

Même si les acteurs du Nevada ont eu une certaine influence sur le débat à l'échelle nationale, la véritable opposition n'est pas venue de leur part (Lobdell, op. cit.). Parfois qualifié de partisans du BANANA, du NIABY ou encore du NOPE, des groupes environnementaux majeurs et bien organisés s'opposent tout simplement à tout développement de la filière nucléaire $^{16}$. Dans le cas de Yucca Mountain, ces groupes sont parvenus, dans un premier temps, à faire contrepoids au puissant lobby des États américains désireux de régler le problème des déchets nucléaires en utilisant le site du Nevada (Idem). De plus, ils sont à l'origine de la stagnation du projet auprès des instances décisionnelles. En effet, en décembre 2004 le projet était toujours sur la glace et ne semblait pas vouloir se réaliser ${ }^{17}$. Cette situation fait dire à plusieurs qu'il est temps de mettre de côté ce projet voué à l'échec et d'envisager d'autres alternatives (Idem). Les groupes de type BANANA, NIABY ou NOPE sont donc parvenus à leurs fins en sabordant le projet de Yucca Mountain. Ce sont donc des groupes organisés et capables (à l'aide de ressources financières, humaines et techniques) de faire contrepoids au promoteur qui ont joué un rôle central dans le déroulement de cette controverse.

\section{Conclusion}

Nous avons été en mesure de constater que le syndrome NIMBY a occupé et occupe toujours une place importante dans l'interprétation des controverses entourant la mise en place d'infrastructures au cours des dernières décennies. Toutefois, la surutilisation du terme NIMBY nous permet d'avancer que certaines controverses ne peuvent tout simplement pas être comprises à travers cette seule notion. Nous avons également mis en lumière que les dynamiques sociales associées au NIMBY semblent avoir évolué et pris la forme de mouvements comme le NOPE, le BANANA ou encore le NIABY. Nous avons qualifié cette évolution de radicalisation sans assise spatiale. Ce changement semble principalement être caractérisé par quatre principaux éléments :

- le passage d'un discours qui se construit autour de l'intérêt individuel à un discours plus axé vers l'intérêt collectif;

- la perte d'importance de la notion de localisation de l'équipement;

- un «approfondissement» des débats entre acteurs;

- une plus grande implication d'acteurs externes à la région touchée.

\section{Notes biographiques}

Étienne Lyrette, B.SC en urbanisme (UQAM), M.SC. Études urbaines (INRS-UCS), est présentement doctorant en études urbaines à l'INRS-UCS. Ses intérêts de recherche sont principalement axés sur les dynamiques sociales entourant la mise en place d'infrastructure énergétique.

Michel Trépanier est professeur-chercheur à l'INRS-UCS et professeur à l'UQTR pour l'institut de recherche sur les PME.

\section{Notes et références}

1 Trom, D. (1999). « De la réfutation de l'effet NIMBY considérée comme une pratique militante ", Revue française de science politique, vol. 49, $\mathrm{n}^{\circ} 1$, février, p. 37.

2 Ibitayo, O.O. et K.D Pijawka. (1999). «Reversing NIMBY : An Assessment of State Strategies for Sitting Hazardous-waste Facilities », Environment and Planning C : Government and Policy, vol. 17, nº 4, août, p. 379-389. 
3 Guay, P.-Y. (1995). Étude exploratoire de l'impact psychosocial d'un projet à risques environnementaux : le cas d'une installation nucléaire, Études et matériaux - document 6, Département d'études urbaines et touristiques, Université du Québec à Montréal, p. 45.

4 Wolsink, M. (2003). « Reshaping the Dutch Planning System : A Learning Process », Environment and Planning A, vol. 35, $\mathrm{n}^{\circ} 4$, avril, p. 705-723; Matejczyk, A.P. (2001). « Why Not NIMBY? Reputation, Neighbourhood Organisation and Zoning Board in a US Midwestern City », Urban Studies, vol. 38, n 3. p. 507-518; Lake, R.W. (1993). "Rethinking NIMBY». Journal of the American Planning Association, vol. 59, $\mathrm{n}^{\circ} 1$, hiver, p. 87-96.

5 Lyrette, É. et M. Trépanier (2004). « Les dynamiques sociales engendrées par l'implantation du parc éolien Le Nordais ", Vertigo, La revue en sciences de l'environnement. [En Ligne], vol. 5, $\mathrm{n}^{\mathrm{o}}$ 1, mai.

http://www.vertigo.uqam.ca/vol5nol/framerevue.html.

6 Lascoumes, P. (1994). L'éco-pouvoir. Environnements et politiques, Paris, La Découverte, p. 232.

7 Callon, M. et J. Law (1982). «On Interests and their Transformation : Enrolment and Counter-enrolment », Social Studies of Science, vol. 12, p. 615-625.

8 Samson, M. (2003). «Les riverains de Cadarache n'ont pas peur de l'atome ». Le Monde, 3 décembre, p. 14.

9 Samson (2003), p. 14.

10 Augereau, J.-F. et P. Le HIR. (2003). « Des écologistes sont hostiles à la construction à Cadarache du réacteur ITER », Le Monde, 6 décembre, p. 26.
11 Simard, J.-G. (2004). «Action collective et revendication d'une infrastructure routière : le cas de la route 175 reliant les régions du Saguenay-Lac-Saint-Jean et de Québec », mémoire de maîtrise, Montréal, Université de Montréal, $72 \mathrm{p}$.

12 Maillebouis, C. (2003). NIMBY ou la colère des lieux. Le cas des parcs éoliens, Natures Sciences Sociétés, Éditions scientifiques et médicales Elsevier. [En ligne] http://www.sciencedirect.com.

13 Retzinger, J.P. (2004). Making Connections : Examining Global and Local Activism in the Scholarship of Kevin Michael DeLuca and Tarla Rai Peterson, University of California, Berkeley. [En ligne] consulté le 6 janvier 2004. http://www.esf.edu/ecn/downloads/ecc02_retzinger.pdf.

14 Greenburg, M. et L. Osafo. (2000). « Secular and Faithbased Organisations as Reliable Information Sources for Residents of Environmentally Stressed Neighbourhoods », Local Environment, vol. 5, no 2, mai, p. 171-189.

15 Lobdell, S. (2002). The Yucca Mountain Repository and the Future of Reprocessing, American Nuclear Society, WISE Intern (Washington Internships for Students of Engineering). [En Ligne] consulté le 15 décembre 2004. http://www.wise-intern.org/journal02/simonlobdell.pdf.

16 Dans ce cas il question de nucléaire, mais ce phénomène de BANANA, NIABY ou de NOPE est perceptible dans plusieurs autres filières sensibles et à risque.

17 Wald, M.L. (2004). «A New Vision for Nuclear Waste », MIT's Magazine of Innovation Technology Review, décembre. p. 38-44. 


\section{Publicité}

Revue PME 